\title{
Improved numerical approach for the time-independent Gross-Pitaevskii nonlinear Schrödinger equation
}

\author{
A. Gammal, ${ }^{1}$ T. Frederico, ${ }^{2}$ and Lauro Tomio ${ }^{1}$ \\ ${ }^{1}$ Instituto de Física Teórica, Universidade Estadual Paulista, 01405-900 São Paulo, Brazil \\ ${ }^{2}$ Departamento de Física, Instituto Tecnológico da Aeronáutica, Centro Técnico Aeroespacial, \\ 12228-900 São José dos Campos, SP, Brazil \\ (Received 5 February 1999; revised manuscript received 13 April 1999)
}

\begin{abstract}
In the present work, we improve a numerical method, developed to solve the Gross-Pitaevkii nonlinear Schrödinger equation. A particular scaling is used in the equation, which permits us to evaluate the wavefunction normalization after the numerical solution. We have a two-point boundary value problem, where the second point is taken at infinity. The differential equation is solved using the shooting method and RungeKutta integration method, requiring that the asymptotic constants, for the function and its derivative, be equal for large distances. In order to obtain fast convergence, the secant method is used. [S1063-651X(99)04608-5]

PACS number(s): 02.60.Lj, 71.10.-w, 03.75.Fi, 11.10.Lm
\end{abstract}

Precise and fast numerical solutions to nonlinear equations have become considerably important in computational physics. So the numerical procedures are relevant, also to be described, when treating such problems, considering the fact that the computation is time consuming. In the present work, we pay attention especially to this problem, proposing an alternative approach to a method recently described in Ref. [1], which was used to solve the Gross-Pitaevskii [2] nonlinear Schrödinger equation (NLSE) for trapped neutral atoms, with positive two-body scattering lengths. In Ref. [3], the NLSE treated in Ref. [1] was extended to a time-dependent one, for both positive and negative two-body scattering lengths, where the Crank-Nicolson algorithm (appropriate for time evolution) was considered. This approach, however, has the disadvantage that it can only reach stable solutions. In case one needs to add other nonlinear terms (of higher order) in the original equation [4], it is not feasible to reach another region of stable solutions if in between there is an unstable region. This implies that it should be appropriate to combine a static method (such as the one used in Ref. [1]) with the method used in Ref. [3] when we are interested in obtain all the stable and unstable solutions and also the corresponding time evolution. So, in this perspective, any improvement of the method considered in Ref. [1] would be relevant.

In the following, we briefly describe the physics related to the NLSE considered in [1] and the numerical procedure used to solve it. Then we present an alternative approach, which can considerably reduce the time needed to search for the solutions and the normalizations.

The nonlinear Schrödinger equation, which describes the condensed wave function in the mean-field approximation, can be written as [1]

$$
\left[-\frac{\hbar^{2}}{2 m} \nabla^{2}+\frac{m}{2} \omega^{2} r^{2}-\frac{4 \pi \hbar^{2}|a|}{m}|\Psi(\vec{r})|^{2}\right] \Psi(\vec{r})=\mu \Psi(\vec{r}),
$$

where $m$ is the mass of a single atom, $\omega$ the angular frequency of the trap, $\mu$ the chemical potential, and $a$ the scat- tering length. In the present approach, as we are more concerned with numerical aspects; for convenience we treat only cases with negative scattering lengths $(a<0)$. [For the numerical considerations, there are no restrictions about the sign of the scattering length, as the solutions with $a>0$ are equally accessible using the same procedure and changing the sign of the nonlinear term in Eq. (1).] Later we also consider the inclusion of a three-body interaction term.

The chemical potential $\mu$ is fixed by the number $N$ of atoms in the condensed state, which is given by the normalization condition

$$
\int d^{3} r|\Psi(\vec{r})|^{2}=N
$$

In Refs. [1] and [3] the NLSE for Bose-Einstein condensates, as given in Eq. (1), was solved numerically. In Ref. [1], the shooting and the Runge-Kutta methods $[5,6]$ were combined. For a given normalization parameter the corresponding dimensionless equation was solved. The asymptotic form of the wave function was renormalized to be equal to the numerical wave function for a sufficiently large distance. The wave-function normalization parameter was increased until the Wronskian of the asymptotic behavior of the numerical and the analytic function change sign.

Next, we present in detail the numerical approach we have used, in order to show the similarities and subtle differences between this approach and the one of Ref. [1]. As we suggest from our experience, such subtle differences in the numerical procedures will reduce considerably the time needed to search for the solutions. We first rewrite Eq. (1) in dimensionless units, in order to makes apparent the physical scales contained in Eq. (1). By rescaling Eq. (1) for the $s$-wave solution, we obtain [4]

$$
\left[-\frac{d^{2}}{d x^{2}}+\frac{1}{4} x^{2}-\frac{|\Phi(x)|^{2}}{x^{2}}\right] \Phi(x)=\beta \Phi(x),
$$


where $x \equiv \sqrt{2 m \omega / \hbar} \quad r, \quad \Phi(x) \equiv \sqrt{8 \pi|a|} \quad r \Psi(\vec{r})$, and $\beta$ $\equiv \mu / \hbar \omega \leqslant 3 / 2$. The normalization for $\Phi(x)$, obtained from Eq. (2), defines a real number $n$ (given as $\left|C_{n l}^{3 D}\right|$ in Ref. [1]) related to the number of atoms $N$ :

$$
\int_{0}^{\infty} d x|\Phi(x)|^{2}=n, \quad \text { where } n \equiv 2 N|a| \sqrt{\frac{2 m \omega}{\hbar}}
$$

We would like to emphasize that, by using this scaling procedure, the numerical solutions for the equation are free of any normalization constraint or other parameter dependence. The parameter $N$, related to the number of particles, was removed from the differential equation and it is not necessary to check Eq. (2) or (4) at all steps of the calculation. The normalization is found a posteriori, using Eq. (4).

The boundary conditions for Eq. (3) are given as [1]

$$
\begin{gathered}
\Phi(0)=0 \quad \text { and }\left.\Phi(x)\right|_{x \rightarrow \infty} \rightarrow \Phi_{\text {asym }}(x), \\
\Phi_{\text {asym }}(x) \equiv C \exp \left[-\frac{x^{2}}{4}+\left(\beta-\frac{1}{2}\right) \ln (x)\right],
\end{gathered}
$$

where $C$ is a constant to be determined. By using the RungeKutta method and starting with a given $\Phi(0)$, the problem is reduced to determining the value of the corresponding derivative $\Phi^{\prime}(0)$, which satisfies the asymptotic condition at infinity. So we are tempted to shoot $[5,6]$ many values for $\Phi^{\prime}(0)$ until we obtain numerically a constant for large distances. At a certain $x_{\max }$ we expect a constant, given by

$$
C_{\Phi} \equiv \Phi_{n u m}(x) \exp \left[\frac{x^{2}}{4}-\left(\beta-\frac{1}{2}\right) \ln (x)\right]
$$

This process is very laborious and difficult to reach precise solutions due to the problem of verifying, for some large $x$, when $C_{\Phi}$ is constant, within the required numerical precision. The way to overcome these difficulties is to consider the asymptotic derivative of $\Phi(x)$, that is,

$\Phi_{\text {asym }}^{\prime}(x)=C\left[-\frac{x}{2}+\left(\beta-\frac{1}{2}\right) \frac{1}{x}\right] \exp \left[-\frac{x^{2}}{4}+\left(\beta-\frac{1}{2}\right) \ln (x)\right]$,

and also determine (numerically) the expression

$$
\begin{aligned}
C_{\Phi^{\prime}} \equiv & \Phi_{\text {num }}^{\prime}(x)\left[-\frac{x}{2}+\left(\beta-\frac{1}{2}\right) \frac{1}{x}\right]^{-1} \\
& \times \exp \left[\frac{x^{2}}{4}-\left(\beta-\frac{1}{2}\right) \ln (x)\right],
\end{aligned}
$$

with $x_{\max }$ such that both Eqs. (8) and (6) are constants. When we are using the correct value of $\Phi^{\prime}(0)$ we also should obtain $C_{\Phi}=C_{\Phi^{\prime}}=C$ for a large enough $x=x_{\max }$.

A useful remark we can make is that when we overestimate the value of $\Phi^{\prime}(0), C_{\Phi}$ increases and $C_{\Phi^{\prime}}$ decreases.
The inverse happens when we underestimate $\Phi^{\prime}(0)$. So this condition is valuable to tune $\Phi^{\prime}(0)$. It corresponds to solving the equation

$$
C_{\Phi}-C_{\Phi^{\prime}}=0
$$

having $\Phi^{\prime}(0)$ as the unknown variable. Substituting Eqs. (6) and (8) into Eq. (9) we recover the expression for the Wronskian $W\left(\Phi_{\text {num }}\left(x_{\text {max }}\right), \Phi_{\text {asym }}\left(x_{\text {max }}\right)\right)=0$ stated in [1]. Equation (9) can be solved by secant method [6]. So we begin with an approximate solution for $\Phi^{\prime}(0)$, as an input to the secant method, where $C_{\Phi}$ and $C_{\Phi^{\prime}}$ are evaluated by the Runge-Kutta method. We should emphasize that, to succeed with such a method, the original guess for $\Phi^{\prime}(0)$ should be not far from the correct value; otherwise the method can lead to the trivial solution $\Phi(x)=0$ or to overflows. In our procedure, for a fixed $\beta, x_{\max }$ was first estimated to be equal to 4.2 and $\Phi^{\prime}(0)$ was used as an initial trial to extend $x_{\max }$ to 5.6 and subsequently to 7.0. Once we find a solution for $\Phi^{\prime}(0)$, for a given $\beta$, we decrease $\beta$ slightly by $\Delta \beta$ using the previous $\Phi^{\prime}(0)$ to find the new $\Phi^{\prime}(0)$. This process allows us to "walk" along $\beta$ values, obtaining the corresponding solutions and results for $n$.

Although the secant method can become unstable under certain conditions, in this case it will not occur, as we explain in the following. We found that the secant method is appropriate, as we can be as near as desired to the solution, starting with a given analytical solution of the corresponding linear Schrödinger equation. So we just need to implement an automatic algorithm routine to make slow variations of $\beta$ and the corresponding slow shift (from the initially zero) of $\Phi^{\prime}(0)$, in order to satisfy the corresponding nonlinear equation. In this way, we are always near the solution, such that the secant method can be applied. We think the same procedure can be generally applied for solitonic equations. The algorithm of the slow variation of $\beta$ (deformation algorithm) does not need an estimation for the derivative of the wave function at $x=0$ [as given in Eq. (3.7) of Ref. [1]] for every solution, except for the first one where we take it near the harmonic oscillator solution. We understand that the analytical approximation given in Ref. [1] to estimate the derivative at $x=0$ is not the most convenient in the present case. Considering that in general such equations are highly nonlinear, the initial guess for the derivative of $\Phi$ at $x=0$ can easily cause overflow when determining the asymptotic constants (Wronskian) at large distances. Our initial guess can be very close to the harmonic oscillator solution, which corresponds to $\Phi^{\prime}(0)=0$, avoiding possible overflows for sufficiently large distances. In our numerical approach, considering that $\beta=1.5$ is a trivial solution of the linear harmonic oscillator, we started with $\beta=1.4$, trial $\Phi^{\prime}(0)=0.6$, and $\Delta \beta=0.02$. For each $\beta$ four to six iterations were necessary in the secant method [6], for each $x_{\max }$.

Our results, for several values of $\beta$, are partially listed in Table I. The solutions with $\beta \leqslant 0.4$ are unstable and not shown in Ref. [3]. However, the solutions with $\beta \geqslant 0.4$ agree well with their results. As one can observe in Table I, the method also can reach solutions with negative chemical potentials $(\beta<0)$. A numerical stability check, which can be 
TABLE I. Numerical solutions for the NLSE including twobody interaction, when the two-body scattering $a$ is negative. We consider that at $x_{\max }=7.0$ we have achieved the asymptotic limit.

\begin{tabular}{cccc}
\hline \hline$\beta$ & $\phi^{\prime}(0)$ & $C$ & $n$ \\
\hline 1.5 & 0 & 0 & 0 \\
1.4 & 0.5448721 & 0.535 & 0.3310 \\
1.2 & 0.9939222 & 0.929 & 0.8597 \\
1.0 & 1.3567267 & 1.187 & 1.2282 \\
0.8 & 1.7022822 & 1.374 & 1.4607 \\
0.6 & 2.0495486 & 1.510 & 1.5839 \\
0.4 & 2.4045809 & 1.608 & 1.6254 \\
0.2 & 2.5851166 & 1.648 & 1.6237 \\
0.0 & 3.1340461 & 1.741 & 1.5632 \\
-1.0 & 4.8924036 & 2.110 & 1.2234 \\
-2.0 & 6.3914678 & 2.995 & 0.9843 \\
\hline \hline
\end{tabular}

done by evolving the static solutions, can easily be followed by using a time-dependent method, such as the CrankNicolson method $[3,6]$.

In Fig. 1 we also show three plots for the chemical potential $\beta$ as a function of $n$, in the case of zero angular momentum. The three plots shown correspond to the lower radial states $\left(n_{r}=0,2,4\right)$ of Eq. (3). The plot labeled with $n_{r}=0$ corresponds to Table I. In the limit of the harmonic oscillator solution, where $n=0$ and the equation is linear, we obtain the usual known solutions.

In Fig. 2 we have another example of the application of the method described here. In this case, we consider the addition of another nonlinear term inside the square brackets of Eq. (3), given by

$$
g_{3} \frac{|\Phi(x)|^{4}}{x^{4}},
$$

which can be directly related to the three-body effects, where $g_{3}$ is the nondimensional strength of the corresponding three-

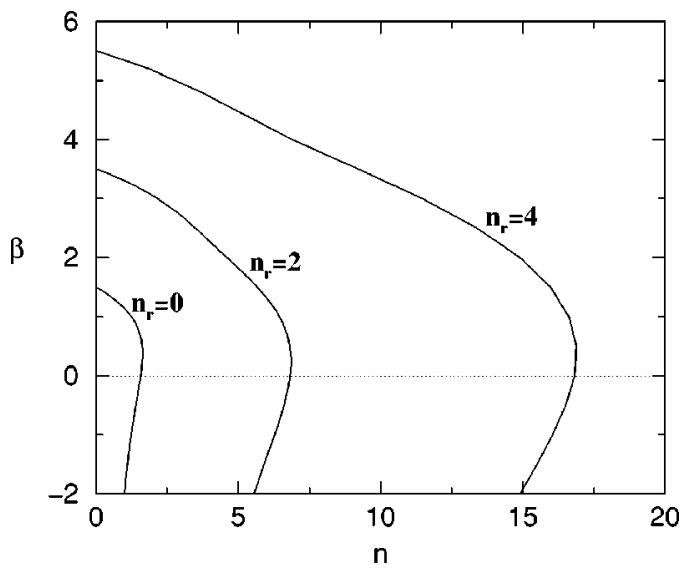

FIG. 1. We show the chemical potential $\beta$ as a function of $n$, which is related to the number of particles by Eq. (4). The three plots shown correspond to the lower radial states $\left(n_{r}\right)$ of Eq. (3) (with zero angular momentum), such that in the limit of the harmonic oscillator solution, where $n=0$, we have the usual known solutions. The plot labeled with $n_{r}=0$ corresponds to Table I.

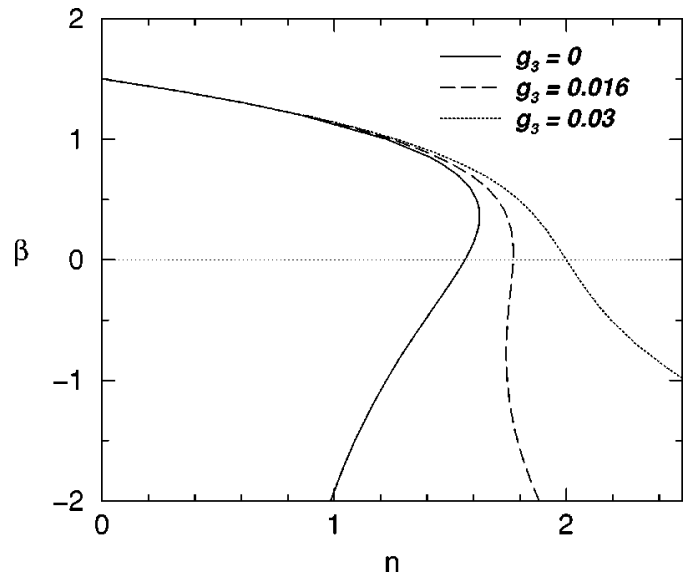

FIG. 2. We show the chemical potential $\beta$ as a function of $n$, in the case where we consider three-body effects. The three plots shown correspond to the radial state solutions for $g_{3}=0$ (no quintic term), $g_{3}=0.016$, and $g_{3}=0.03$. The plot labeled with $g_{3}=0$ corresponds to Table I.

body interaction. The physical consequences of the addition of such a term in the NLSE is discussed in both references given in [4].

To finalize, we have presented in detail an improvement to a numerical procedure used to solve a nonlinear differential equation, which is commonly applied for Bosecondensed states. In our example, we solve the GrossPitaevskii equation with an attractive two-body and a repulsive three-body interaction. We should note that, by using a simplified scaling procedure [given in Eqs. (3) and (4)], the numerical solutions for the equation are free of any normalization constraint or other parameter dependence. The parameter $N$, related to the number of particles, was removed from the differential equation and it is not necessary to obtain the normalization at each step of the calculation. Equation (4) gives the normalization a posteriori. So, by using the above scaling procedure, it emerges that the main differences between the present method and the one given in Ref. [1], when looking for solutions of the NLSE, are that (i) in our approach, we searched for the derivative of the wave function at $x=0$ until the asymptotic constants match (when the Wronskian vanishes) and the normalization is given at the end; (ii) in [1] the normalization parameter $A$ is incremented until the sign of the Wronskian is changed. For the final renormalization they also use other intermediate parameters, such as $A_{0}, N_{0}, A_{1}$, and $N_{1}$.

As we are not restricted by the normalization, our approach is a clear improvement to the method given in Ref. [7], particularly when considering the simplification and the transparency in the normalization procedure. Such an advantage can be further explored when more involved calculations are presented, as in Ref. [7] where collective excitations are evaluated. A different scaling removes the normalization constraint and allows one to obtain it a posteriori, after numerical solutions are achieved for the eigenvalues.

In our numerical procedure, we employ the shooting method on Runge-Kutta integration, matching the asymptotic constants for the wave function and for the corresponding 
derivative. This procedure is shown to be equivalent to making the Wronskian vanish at such large distances. In order to obtain a faster convergence to the solution, we also included the secant method. The numerical optimization of the method employed in Ref. [1], described here, is not restricted to the NLSE we have used. It can be used quite generically for second-order solitonic differential equations whose solutions asymptotically vanish at large distances. We consider particularly relevant an optimization of the method of Ref.
[1] from the perspective of looking for solutions of differential equations with higher-order nonlinear terms and also from the perspective of combining such a method (appropriate for static solutions) with a time-dependent one.

This work was partially supported by the Fundação de Amparo à Pesquisa do Estado de São Paulo and Conselho Nacional de Desenvolvimento Científico e Tecnológico.
[1] M. Edwards and K. Burnett, Phys. Rev. A 51, 1382 (1995).

[2] V.L. Ginzburg and L.P. Pitaevskii, Zh. Éksp. Teor. Fiz. 34, 1240 (1958) [Sov. Phys. JETP 7, 858 (1958)]; L.P. Pitaevskii, ibid. 40, 646 (1961) [ibid. 13, 451 (1961)]; E.P. Gross, J. Math. Phys. 4, 195 (1963).

[3] P.A. Ruprecht, M.J. Holland, K. Burnett, and M. Edwards, Phys. Rev. A 51, 4704 (1995).

[4] A. Gammal, T. Frederico, and L. Tomio, in Proceedings of the International Workshop on Collective Excitations in Fermi and Bose Systems, Serra Negra, Brazil, 1998, edited by C. Bertulani, L. F. Canto, and M. Hussein (World Scientific, Sin- gapore, 1999); A. Gammal, T. Frederico, L. Tomio, and Ph. Chomaz, e-print cond-mat/9904184.

[5] W.H. Press, S.A. Teulkolsky, W.T. Vettering, and B.P. Flannery, Numerical Recipes in Fortran, 2nd ed. (Cambridge University Press, Cambridge, England, 1992).

[6] D. Quinney, An Introduction to the Numerical Solution of Differential Equations, revised ed. (Wiley, New York, 1987), p. 133.

[7] M. Edwards, P.A. Ruprecht, K. Burnett, R.J. Dodd, and C.W. Clark, Phys. Rev. Lett. 77, 1671 (1996). 